\title{
BOUNDARIES OF THE SPECTRA IN $\mathscr{L}(X)$
}

\author{
WOO YOUNG LEE
}

(Communicated by Palle E. T. Jorgensen)

\begin{abstract}
Suppose $T$ is a bounded linear operator on a complex Banach space $X$. If $T$ is "regular" with some finite dimensional intersection property and if 0 is in the boundary of the spectrum of $T$ then 0 is an isolated point of it.
\end{abstract}

In [8], Putnam proved that if $T$ is a bounded linear operator on a Hilbert space and if $\lambda$ is an accumulation point of the boundary of the spectrum of $T$ then either the range of $T-\lambda I$ is not closed or the dimension of the null space of $T-\lambda I$ is infinite.

In this note we generalize this result in the case of a Banach space.

Throughout this note suppose $X$ and $Y$ are complex Banach spaces, write $\mathscr{L}(X, Y)$ for the set of bounded linear operators from $X$ to $Y$, and abbreviate $\mathscr{L}(X, X)$ to $\mathscr{L}(X)$. If $T \in \mathscr{L}(X)$ then we write $\alpha(T)$ and $\beta(T)$ for the $\operatorname{dim} T^{-1}(0)$ and $\operatorname{codim} T(X) ; \sigma(T)$ for the spectrum of $T$. If $K$ is a compact subset of the complex plane $\mathbf{C}$, write $\partial K$ and iso $(K)$, respectively, for the topological boundary points and the isolated points of $K$.

Recall that an operator $T \in \mathscr{L}(X, Y)$ is regular if there is $T^{\prime} \in \mathscr{L}(Y, X)$ for which

$$
T=T T^{\prime} T .
$$

If $(0.1)$ holds then $T^{\prime}$ is called a generalized inverse for $T$. It is well known that $T$ is regular if and only if $T(X)$ is closed and both $T^{-1}(0)$ and $T(X)$ are complemented (cf. [5,6]). Recall, also, that $T \in \mathscr{L}(X, Y)$ is upper semiFredholm if $T(X)$ is closed and $\alpha(T)<\infty$ and is lower semi-Fredholm if $T(X)$ is closed and $\beta(T)<\infty$. If $T$ is upper or lower semi-Fredholm it is called semi-Fredholm.

We begin with:

Lemma 1. Suppose $X$ is a Banach space and $X=Y \oplus Z$. If $Y^{\prime}$ is a closed subspace of $X$ for which

$$
Y^{\prime} \cong Y \quad \text { and } \quad Y^{\prime} \cap Z \text { is finite dimensional }
$$

then $Y^{\prime}+Z$ is closed in $X$.

Received by the editors October 10,1990 and, in revised form, February 21, 1991.

1980 Mathematics Subject Classification (1985 Revision). Primary 47A10.

Key words and phrases. Regular operator, punctured neighborhood theorem, spectrum, essential spectrum, Weyl spectrum.

Research supported in part by a grant from KOSEF in 1990.

(c) 1992 American Mathematical Society $0002-9939 / 92 \$ 1.00+\$ .25$ per page 
Proof. Suppose that $X=Y \oplus Z, Y^{\prime} \cong Y$, and $P=P^{2} \in \mathscr{L}(X)$ with $Z=$ $P(X)$. If $Y^{\prime} \cap Z$ is finite dimensional then we can find a closed subspace $Y_{0}$ for which

$$
\left(Y^{\prime} \cap Z\right) \oplus Y_{0}=Y^{\prime} .
$$

Also, choosing subspaces $W$ and $W_{0}$ of $Y$ in such a way that

$$
Y^{\prime} \cap Z \cong W \quad \text { and } \quad W \oplus W_{0}=Y,
$$

then we have $W_{0} \cong Y_{0}$ because $Y^{\prime} \cong Y$; therefore $Z \oplus W_{0} \cong Z \oplus Y_{0}$. We then can find a projection $Q=Q^{2} \in \mathscr{L}(Y)$ with $W_{0}=Q(Y)$. We now claim that

$$
P+Q(I-P): X \rightarrow X
$$

is a continuous projection on $X$ : indeed, for each $x=y+z \in X$ with $y \in Y$ and $z \in Z$,

$$
(P+Q(I-P))(x)=P(y+z)+Q(I-P)(y+z)=z+Q(y)
$$

and

$$
(P+Q(I-P))^{2}(x)=(P+Q(I-P))(z+Q(y))=z+Q(Q(y))=z+Q(y) .
$$

Therefore it follows at once that

$$
(P+Q(I-P))(X)=Z \oplus W_{0} \cong Z \oplus Y_{0}=Z+Y^{\prime}
$$

is closed in $X$. The last equality of (1.2) comes from (1.1) and the observation that

$$
\begin{aligned}
x \in Z+Y^{\prime} & \Rightarrow x=z+y_{1}+y_{2} \quad \text { with } z \in Z, y_{1} \in Y^{\prime} \cap Z, \text { and } y_{2} \in Y_{0} \\
& \Rightarrow x \in Z+Y_{0} .
\end{aligned}
$$

Our main theorem is a generalization of the "punctured neighborhood theorem":

Theorem 2. Let $X$ be a Banach space and let $T \in \mathscr{L}(X)$ be regular, with generalized inverse $T^{\prime} \in \mathscr{L}(X)$. Suppose that $S \in \mathscr{L}(X)$ is invertible and commutes with $T$, and also satisfies that $I-S T^{\prime}$ is invertible. If

$$
T(X) \cap T^{-1}(0) \quad \text { and } \quad\left(I-S T^{\prime}\right) T(X) \cap T^{-1}(0)
$$

are finite dimensional then

$$
T-S \text { is upper semi-Fredholm }
$$

and hence

$$
0 \in \partial \sigma(T) \Rightarrow 0 \in \text { iso } \sigma(T) \text {. }
$$

Proof. Suppose $T=T T^{\prime} T$ is regular; thus $T^{\prime} T(X)$ is the complementary subspace to $T^{-1}(0)$. Writing

$$
M=(T-S) T^{\prime} T(X) \cap T^{-1}(0),
$$

then, by assumption, $M$ is finite dimensional. Observe that, by assumptions,

$$
(T-S) T^{\prime} T(X) \cong T(X) \cong T^{\prime} T(X) \quad \text { and } \quad S T^{-1}(0)=T^{-1}(0) \text {. }
$$


We now claim that

$$
T-S \text { is upper semi-Fredholm. }
$$

Indeed, since $\operatorname{dim} M<\infty$, it follows from (2.4) that

$$
\begin{aligned}
(T-S)(X) & =(T-S)\left(T^{\prime} T(X) \oplus T^{-1}(0)\right) \\
& =(T-S) T^{\prime} T(X)+T^{-1}(0),
\end{aligned}
$$

which is closed in $X$, because the conditions of Lemma 1 are satisfied with $T^{-1}(0)$ in place of $Z$ and $(T-S) T^{\prime} T(X)=Y^{\prime}$ and that for each $x=y+z$ with $y=T^{\prime} T(w) \in T^{\prime} T(X)$ and $z \in T^{-1}(0)$,

$$
\begin{aligned}
x \in(T-S)^{-1}(0) \Rightarrow & (T-S)\left(T^{\prime} T(w)\right)-S(z)=0 \\
\Rightarrow & \left(I-S T^{\prime}\right) T(w) \in M \text { and } S(z) \in M \\
\Rightarrow & T(w) \in\left(I-S T^{\prime}\right)^{-1}(M) \text { and } z \in S^{-1}(M) \\
\Rightarrow & x \in G+H \\
& \quad \text { with } G=T^{\prime}\left(I-S T^{\prime}\right)^{-1}(M) \text { and } H=S^{-1}(M),
\end{aligned}
$$

where the second implication uses the second equality of (2.4) and $G+H$ must be finite dimensional; thus $\alpha(T-S)$ is finite. This proves (2.2).

Toward (2.3), apply the punctured neighborhood theorem to $T-S$ with $S=\mu I$ and $0<|\mu|<\delta$; then $\alpha(T-\lambda I)$ and $\beta(T-\lambda I)$ are constant on a punctured neighborhood of 0 (see [3, Theorem V.1.8]). If $0 \in \partial \sigma(T)$ then it follows that for some $\theta$ with $0<\theta<\delta, \alpha(T-\lambda I)=\beta(T-\lambda I)=0$ for $0<|\lambda|<\theta$; thus $T-\lambda I$ is invertible for $0<|\lambda|<\theta$, giving (2.3).

We have been unable to decide whether or not the second condition of (2.1) can be dropped from Theorem 2. We conjecture that this is affirmative; we have not yet found a counterexample.

A semi-Fredholm operator $T$ is Fredholm if both $\alpha(T)$ and $\beta(T)$ are finite. The essential spectrum of $T$, denoted $\sigma_{e}(T)$, is defined by

$$
\sigma_{e}(T)=\{\lambda \in C: T-\lambda I \text { is not Fredholm }\} .
$$

If $T \in \mathscr{L}(X)$ is semi-Fredholm then the index of $T$ is defined by

$$
\operatorname{index}(T)=\alpha(T)-\beta(T) \text {. }
$$

An operator $T \in \mathscr{L}(X)$ is Weyl if $T$ is Fredholm and $\operatorname{index}(T)=0$. The Weyl spectrum of $T$, denoted $\sigma_{w}(T)$, is defined by

$$
\sigma_{w}(T)=\{\lambda \in C: T-\lambda I \text { is not Weyl }\} .
$$

Both $\sigma_{e}(T)$ and $\sigma_{w}(T)$ are compact (cf. $[1,2,5,6]$. If we define

$$
P_{n}(T)=\{\lambda \in \sigma(T): T-\lambda I \text { is semi-Fredholm and index }(T-\lambda I)=n\},
$$

where $n \in Z \cup\{ \pm \infty\}$, then we can write

$$
\sigma_{w}(T)=\sigma_{e}(T) \cup\left\{\bigcup_{n \neq 0} P_{n}(T)\right\} .
$$

For the essential spectrum and the Weyl spectrum, we can obtain the analogue of (2.3): 
Theorem 3. If $X$ and $T$ satisfy the hypotheses in Theorem 2, then there is implication

$$
0 \in \partial \sigma_{e}(T) \Rightarrow 0 \in \text { iso } \sigma_{e}(T) \quad \text { and } \quad 0 \in \partial \sigma_{w}(T) \Rightarrow 0 \in \text { iso } \sigma_{w}(T) .
$$

Proof. By the same argument as Theorem 2, $(T-\lambda I)(X)$ is closed and both $\alpha(T-\lambda I)$ and $\beta(T-\lambda I)$ are constant on a punctured neighborhood of 0 . Since $0 \in \partial \sigma_{e}(T)$, it follows that for some $\delta>0$, both $\alpha(T-\lambda I)$ and $\beta(T-\lambda I)$ are finite for $0<|\lambda|<\delta$. Thus we have $0 \in$ iso $\sigma_{e}(T)$, giving the first implication of (3.1).

Toward the second implication, observe that for each $n \in Z \cup\{ \pm \infty\}$ with $n \neq 0, P_{n}(T)$ is a hole of $\sigma_{e}(T)$; thus by (2.5) the passage from $\sigma_{e}(T)$ to $\sigma_{w}(T)$ is the filling in certain of its holes; therefore we have $\partial \sigma_{w}(T) \subseteq \partial \sigma_{e}(T)$. This together with the first implication gives the result.

An operator $T \in \mathscr{L}(X, Y)$ is decomposably regular if there is $T^{\prime} \in \mathscr{L}(Y, X)$ for which

$$
T=T T^{\prime} T \quad \text { and } \quad T^{\prime} \text { is invertible. }
$$

It is well known that $T$ is decomposably regular if and only if

$$
T \text { is regular and } T^{-1}(0) \cong Y / T(X) .
$$

Also if $T \in \mathscr{L}(X)$ then we have

$$
\begin{aligned}
& \{T \in \mathscr{L}(X): T \text { decomposably regular }\} \\
& \quad=\{T \in \mathscr{L}(X): T \text { regular }\} \cap \operatorname{cl}\{T \in \mathscr{L}(X): T \text { invertible }\} .
\end{aligned}
$$

This follows from the result of Harte [4], wherein can be found a generalization. As a special case of Theorem 2, we have:

Corollary 4. Let $X$ be a Banach space. If $T \in \mathscr{L}(X)$ is regular and if at least one of $\alpha(T)$ and $\beta(T)$ is finite then there is implication

$$
0 \in \partial \sigma(T) \Rightarrow 0 \in \text { iso } \sigma(T) .
$$

Proof. Suppose $0 \in \partial \sigma(T)$ but $T$ is regular; thus by (3.3) $T$ is decomposably regular. If $\alpha(T)<\infty$ then trivially the condition (2.1) is satisfied; thus it follows from Theorem 2 that $0 \in$ iso $\sigma(T)$.

If instead $\beta(T)<\infty$ then by (3.2) we also have $\alpha(T)<\infty$. This completes the proof.

We might remark that, remembering that if $T \in \mathscr{L}(X)$ then

$$
T \text { Fredholm } \Rightarrow T \text { regular, }
$$

it clearly follows from Corollary 4 that

$$
\partial \sigma(T) \backslash \sigma_{e}(T) \subseteq \text { iso } \sigma(T)
$$

(see $[5,(9.8 .4 .1)])$; consequently $(4.2)$ is a special case of Theorem 2 . 
If $X$ is a Hilbert space and $T \in \mathscr{L}(X)$ then

$$
T \text { regular } \Leftrightarrow T(X) \text { closed; }
$$

thus in this case Corollary 4 reduces to [2, Theorem XI.5.3].

\section{ACKNOWLEDGMENT}

I wish to express my appreciation to the referee whose suggestions led to an improvement of the paper.

\section{ADDED IN PROOF}

We have recently shown that the second condition of (2.1) can be dropped from Theorem 2 (W. Y. Lee, A generalization of the punctured neighborhood theorem, Proc Amer. Math. Soc. (to appear)).

\section{REFERENCES}

1. S. K. Berberian, The Weyl spectrum of an operator, Indiana Univ. Math. J. 20 (1970), 529-544.

2. J. B. Conway, A course in functional analysis, Springer-Verlag, New York, 1985.

3. S. Goldberg, Unbounded linear operators, McGraw-Hill, New York, 1966.

4. R. E. Harte, Regular boundary elements, Proc. Amer. Math. Soc. 99 (1987), 328-330.

5. _ Invertibility and singularity for bounded linear operators, Marcel Dekker, New York, 1988.

6. H. G. Heuser, Functional analysis, Wiley, New York, 1982.

7. W. Y. Lee, Relatively open mappings, Proc. Amer. Math. Soc. 108 (1990), 93-94.

8. C. R. Putnam, The spectra of operators having resolvents of first order growth, Trans. Amer. Math. Soc. 133 (1968), 505-510.

9. W. Rudin, Functional analysis, McGraw-Hill, New York, 1973.

10. A. Wilansky, Modern methods in topological vector spaces, McGraw-Hill, New York, 1978.

Department of Mathematics, Sung Kyun Kwan University, Suwon 440-746, Korea 